\title{
ESTUDIO FUNCIONAL DE ÚTILES PULIMENTADOS: EXPERIMENTO DE TALA Y ANÁLISIS DE MICRODESGASTE
}

\author{
FUNCTIONAL STUDY OF POLISHED TOOLS. AN EXPERIMENT \\ IN TREE-CHOPPING AND MICRO-WEAR ANALYSIS
}

\author{
por \\ RAMON FABREGAS VALCARCE
}

RESUMEN Se llevó a cabo un primer ensayo de tala de árboles (aliso) con un hacha de piedra pulida, la cual efectuó la tarea con gran eficiencia y sin que se apreciase un desgaste significativo. El experimento hubo de interrumpirse debido a problemas con la fijación del hacha al mango de madera. Asimismo se ha realizado un examen de microdesgaste sobre 5 microhachas, hallándose indicios de que una parte de ellas pudo ser utilizada en una dinámica de tipo azuela, mientras que un ejemplar (n.. 5) sería empleado tal vez en tareas de raspado o cepillado. En conjunto, el trabajo experimental y las observaciones efectuadas señalan la necesidad de una discriminación más precisa entre las alteraciones debidas al uso y aquéllas originadas en el curso de la elaboración de las piezas.

SUMMARY Functional study of ground-stone tools: A tree-felling experiment and microwear analysis. In this paper we carry on a limited experiment of tree felling (alder) with a polished axe (fig. 1). The tool was shown to be quite efficient although problems with hafting prevented us from continuing the task further. The axe blade was submitted to microscopical examination prior and after the experiment, with no significant damage on the working edge reported. Also we have looked for eventual wear traces on 5 'microaxes' (fig. 2), the result suggesting that some of them might have been used as adzes, while another (. .05$)$ was perhaps employed for planing or scraping. All in all, the experiment and microscopical observations point to the need of making a more objective distinction between polishes or striations provoked by use and those due to manufacture.

\section{INTRODUCCION}

Desde casi la fundación de la Prehistoria como disciplina hubo intentos de averiguar la función o probar la eficacia de los artefactos arqueológicos. Con este fin se recurrió a los paralelos etnográficos, al sentido común (a veces de forma poco juiciosa) y a la realización de experimentos. 
Entre los pioneros en la experimentación con hachas de piedra pulida hay que mencionar a $\mathrm{N}$. Sehested (1884), que a fines del siglo pasado en Dinamarca, fabricó, enmangó y utilizó instrumentos pulimentados para talar y trabajar la madera. En la misma línea P. Quente (1914) en Alemania realizó un ambicioso esfuerzo en el que se combinan la experimentación con la observación mediante lupa de las huellas de uso sobre útiles pulimentados de corte. En los años cincuenta se acometieron proyectos en los que la utilización de artefactos de piedra pulida formaba parte de investigaciones más amplias sobre antiguas técnicas agrícolas, entre las que destacan las llevadas a cabo en Draved (Dinamarca) (Iversen, 1956; Jørgensen, 1985) y, posteriormente, en Durham (Inglaterra) (Harding y Young, 1979). Otros trabajos orientados a elucidar algunos problemas específicos son los de Sonnenfeld (azadas) (1962), Sillitoe (rendimiento comparado de hachas de acero y piedra pulida) (1979) u Olausson (comparación entre hachas de sílex y otros tipos de roca) (1982 y 1982-83).

En esas y otras experiencias con reproducciones de hachas u otro tipo de útiles pulimentados, el estudio complementario de las huellas de uso ha sido comparativamente escaso (Olausson 1982; 1982 83, 56-58; Sonnenfeld, 1962) y los análisis de microdesgaste sobre artefactos de piedra pulida se han limitado generalmente a la detección de estrías u otros restos de uso sólo sobre materiales arqueológicos, interpretando funcionalmente la disposición y características del desgaste de éstos a la luz de paralelos etnográficos o simplemente de la "lógica". Semenov en su conocido estudio recurre fundamentalmente, aunque no en exclusiva, a la comparación con herramientas metálicas actuales cuando estudia la función de distintos útiles pulimentados (Semenov, 1981, 237). El método de Semenov ha tenido muchos seguidores, como Detev (1960), Kantman (1969-70), Born (1971), Delibes (1974) o Roodenberg (1982), si bien algunos autores han mostrado su escepticismo, basándose fundamentalmente en la dificultad de distinguir las estrías producidas por el pulimento o posteriores reafilados, de aquéllas otras específicamente originadas por la utilización (Cauvin, 1968, 24; Stelcl y Malina, 1970, 52; Kantman, 1969-70, 145), mientras otros investigadores critican el subjetivismo que impregna a algunas definiciones del microdesgaste sobre herramientas de piedra pulida (Keeley, en Roodenberg, 1982, 187; Madsen, 1984, 56).

Con el presente trabajo pretendemos efectuar un análisis funcional sobre dos clases concretas de utensilios pulimentados. Por una parte, hemos procedido a efectuar un primer ensayo de tala de árboles con un hacha que por sus características morfométricas encaja dentro de una variedad común (tipo II, Fábregas, 1988,22) en contextos tumulares o domésticos del Noroeste, observando a continuación las huellas de desgaste macro o microscópicas que dicha tarea pudiese producir eventualmente. Por otro lado, hemos manejado una muestra de 5 microhachas, útiles de muy pequeñas dimensiones que constituyen un segmento importante dentro del grupo de pulimentados procedentes de monumentos megalíticos en el Noroeste (Fábregas, 1991, 70). Las piezas aquí estudiadas tienen una proveniencia diversa y han sido sometidas a un exhaustivo examen microscópico con el fin de detectar cualquier tipo de evidencias (microfracturas, estrías, abrasión) indicativas de un empleo determinado.

Todas las observaciones microscópicas fueron efectuadas con un microscopio binocular de luz incidente, Olympus Vanox (empleando objetivos entre 50x y 200x), y las fotos realizadas mediante el sistema microfotográfico Olympus PM-10AD, señalándose sobre la figura 2 los puntos donde se tomaron éstas. Estas investigaciones fueron llevadas a cabo en el curso de una estancia de un año en el Instituto de Arqueología de la Universidad de Londres, subvencionada mediante una beca Fleming !

1. Quiero expresar mi agradecimiento al profesor Dr. J. M. Caamaño Gesto y al Dr. J. C. Valle Pérez, del Departamento de Prehistoria y Arqueología de la Universidad de Santiago y Museo de de Pontevedra, respectivamente, por las facilidades otorgadas para el estudio de estos objetos. Este trabajo no hubiera sido posible sin la colaboración y consejo aportados por el profesor Dr. Mark H. Newcomer y el Dr. Roger Grace, adscritos al Departamento de Prehistoria del Instituto de Arqueología. 


\section{A. Experimento de tala}

La pieza utilizada está hecha de una roca (greenstone) formada a partir de un proceso de metamorfismo regional. Sus características morfológicas son las siguientes:

Longitud: $118 \mathrm{~mm}$. Anchura: $64 \mathrm{~mm}$. Espesor: $24 \mathrm{~mm}$.

Peso: 265 grs. L/A: 1'8; A/E:2'6; L/E: 4'9.

Forma general: trapezoidal. Caras: curvas convexas.

Lados: rectos. Aristas: convergentes y curvilíneas.

Filo: convexo simétrico. Zona activa: convexa simétrica.

Talón: redondeado y apuntado. Sección transversal: biconvexa. Acabado: pulimentado integral total.

Clasificación: de acuerdo con los parámetros enunciados podría encuadrarse dentro de las hachas de tipo II.

El hacha fue elaborada por John Lord, ${ }^{2}$ el cual invirtió media hora en lograr la forma general del artefacto mediante percusión. A continuación se procedió al picoteado y pulimento de aquél, utilizando como agentes abrasivos arena y agua, tareas que requirieron un total de seis horas. Estas cifras no difieren significativamente de las documentadas en otros experimentos sobre materiales similares (Olausson, 1982-83, 28; Harding, 1983, 41).

El mango del hacha estaba hecho en madera de fresno y tenía una longitud total de $609 \mathrm{~mm}$. por una anchura máxima de $58 \mathrm{~mm}$. y un grosor también máximo de $44 \mathrm{~mm}$., estas dos últimas magnitudes fueron tomadas en el punto donde se había practicado un orificio, de $61 \mathrm{~mm}$. de profundidad, destinado a encajar el hacha; el peso del mango era de 464 grs. Fue realizado por el Dr. M.H. Newcomer en un tiempo de cuatro horas recurriendo para ello a herramientas metálicas y tanto la madera empleada como el diseño general se corresponden con modelos arqueológicos recuperados en el Centro y Norte de Europa (Becker, 1949, figs. 1-5; Müller-Beck, 1965, figs. 1-3; Jørgensen, 1985, fig. 11). El hacha fue introducida en el alvéolo y fijada dentro de éste mediante una combinación de resina de pino y cera de abejas (fig. 1).

El árbol escogido para la tala fue el aliso europeo (Alnus glutinosa) (gallego: ameneiro), especie de hoja perenne presente en toda la Europa atlántica, en tierras húmedas, con frecuencia en las márgenes de los ríos, de porte rectilíneo, alcanzando unos $10 \mathrm{~m}$. de altura por término medio. Su madera es de dureza mediana y resistente a la humedad, siendo preferida en Galicia para la fabricación de los tradicionales zuecos, pero también usada en la elaboración de muebles, para cerrar las fincas o simplemente como combustible.

El experimento tuvo lugar en una finca particular del país de Gales durante un día del mes de Junio de 1985, con una temperatura atmósferica en torno a los $18^{\circ}$ y gran humedad. Los objetivos de aquél fueron tres fundamentalmente:

A. Probar la eficacia de un hacha del tipo II.

B. Observar si se producía un gran desgaste a nivel macroscópico.

C. Intentar localizar restos microscópicos de usura: estrías o pulimento.

El corte de los árboles fue practicado a una altura de aproximadamente $1 \mathrm{~m}$. sobre el nivel del suelo y la técnica utilizada fue la recomendada por distintos expertos (Müller-Beck, 1965, 179; Harding y Young, 1979, 104; Jørgensen, 1985, 30), es decir un golpe administrado no con toda la fuerza del hombro como se realizaría con un hacha de acero, sino actuando con la articulación del codo; por otra

2. Especialista en tecnología lítica asignado al Museo de Grime's Graves (Norfolk, Inglaterra). 
parte la hoja debe golpear el tronco en un ángulo lo más agudo posible, extrayendo en consecuencia virutas alargadas (fig. 3,1). Tan sólo se cronometró el tiempo de trabajo efectivo y no el empleado en descansar.

Experimento n.․ 1.- Se corta un tronco de $12 \mathrm{~cm}$. de diámetro en 61 minutos. El árbol cae cuando el tajo había penetrado $8 \mathrm{~cm}$. en línea recta y se habían practicado algunos cortes complementarios a los lados (en esta última tarea se invirtieron 5 minutos) (fig. 4,1). No se observa desgaste en el filo del hacha a simple vista.

Experimento n.․ 2.- Se corta un tronco de $18 \mathrm{~cm}$. de diámetro en 60 minutos. El árbol cae cuando el hacha había penetrado a una profundidad de sólo $7 \mathrm{~cm}$. y tras haberse practicado cortes laterales durante cinco minutos. La razón de esta mayor rapidez en relación con el experimento anterior estriba en que el tronco presentaba una inclinación natural que ayudaba a derribarlo una vez que el corte había alcanzado esas dimensiones, simplemente con la fuerza conjunta de dos hombres (fig. 3,2). El hacha todavía presentaba el filo en perfectas condiciones.

Experimento n.․ 3.- Se comienza el corte de un tronco de $15 \mathrm{~cm}$. de diámetro. A los 13 minutos del comienzo el hacha se sale del alvéolo, observándose una grieta en este último. Se intenta reparar el útil fijándolo mediante una cuña de madera con lo que puede continuarse la labor durante otros 3 minutos más. Cuando se abandona el experimento el corte había progresado $6 \mathrm{~cm}$. en el interior del árbol (fig. 9,3). El hacha seguía sin mostrar un desgaste aparente aunque sí se observaba un tenue lustre en las áreas inmediatas al filo.

A la hora de valorar la eficiencia del hacha de piedra pulida hay que tener en cuenta varios factores que inciden en el resultado final del experimento: en primer lugar lo limitado de éste debido a problemas con el enmangue; en segundo lugar la inexperiencia del "leñador" que explica lo dilatado de los tiempos requeridos para acometer la tarea, tiempos que fueron significativamente reducidos en el tercer experimento $(6 \mathrm{~cm}$. en $16 \mathrm{~min}$., o sea 2'6 minutos por centímetro, media que en el primer experimento era de 7 minutos por centímetro aproximadamente). La incidencia que en el desarrollo de este tipo de experimentos tiene el factor experiencia y familiaridad con las particularidades mecánicas y tecnológicas del hacha de piedra ha sido señalada entre otros por Sillitoe $(1979,151)$ o Harding y Young $(1979,104)$.

Hay que destacar que el desgaste visible a nivel macroscópico fue muy escaso y en cualquier caso menor del previsto a tenor de las características de la pieza. Desde luego se puede alegar justificadamente que la ausencia de desgaste carece de significación dado el breve tiempo que el hacha fue utilizada. Sin embargo, en otros experimentos con hachas de piedra pulida (diorita y anfibolita), si bien empleadas sobre madera más dura (roble), se produjo una significativa abrasión del filo al cabo de un corto espacio de tiempo (entre 5 y 21 minutos de trabajo) (Olausson, 1982-83, 42 y 44). Por otra parte, se podría esperar que la inexperiencia del que suscribe en este tipo de tareas provocaría un mayor uso del filo debido a un impulso excesivo o a errores en el ángulo de incidencia del hacha sobre el tronco.

En el curso del presente experimento se ha puesto de manifiesto la importancia que un adecuado enmangue tiene para la consecución de una herramienta eficiente. En nuestro caso hubiese sido necesario probablemente un mayor grosor de la zona del mango donde se insertaba el hacha con la finalidad de evitar el astillado del alvéolo. A este respecto, Harding y Young $(1979,104)$ plantean la conveniencia de que los anillos de la madera que constituye el mango se dispusiesen de forma ortogonal respecto al plano del alvéolo, aunque la evidencia de Seeberg parece indicar lo contrario (Olausson, 1982, 44). Otra posibilidad para evitar la rotura del mango es la apuntada por Jørgensen $(1985,25)$, haciendo que sólo los lados del hacha estén en contacto estrecho con la vaina, dejando un espacio entre esta última y las caras de la pieza. 
La acción del hacha configuró sobre el tronco del árbol un corte de una forma muy peculiar, con un perfil transversal en triángulo rectángulo, estando la base en la parte inferior del tajo (fig. 3,2). Esta particular disposición es muy similar a la documentada en postes recuperados en Burgäschisee-Süd (Suiza) (Müller-Beck, 1965, 130 y fig. 276). La razón para que los cortes practicados en el árbol sean tan abiertos estriba en la necesidad de evitar que el hacha quede atascada en el tajo con el consiguiente peligro de fractura de aquélla.

\section{Estudio microscópico del desgaste sobre el hacha.}

Con anterioridad a su utilización el hacha fue examinada bajo el microscopio, aunque con cierta dificultad debido a que el enmangue hacía incómoda su colocación en el porta. Utilizando entre 50x y 100x se pudo observar la existencia de fínas estrías perpendiculares al filo prolongándose más alla de la zona activa (foto C, fig. 4,$2 ; 100 x$ ). A todo lo ancho de la superficie de la pieza destacaban pequeñas áreas pulimentadas (foto $\mathrm{F}$, fig. 4,$3 ; 50 \mathrm{x}$ ) y el filo aparecía en buen estado, con sólo algunas microlascas visibles (foto $\mathrm{G}$, fig. 5,$1 ; 50 \mathrm{x}$ ).

Una vez utilizada, se observaba a simple vista la presencia de residuos orgánicos (savia o pequeñas fibras leñosas) en un área que abarcaba hasta unos $35 \mathrm{~mm}$. de distancia del filo. Por otra parte, se detectaba una zona lustrosa yendo a lo largo del filo en una banda con un mayor desarrollo hacia uno de los vértices de éste, donde alcanzaba unos $7 \mathrm{~mm}$. de anchura; este lustre era más patente en una de las caras (B) (figs. 2 y 5,2).

El examen microscópico fue problemático por dos circunstancias: el tamaño de la pieza, excesivo para trabajar con holgura, y la incurvación de la superficie del hacha, lo cual hace muy difícil la obtención de fotos nítidas, incluso con pocos aumentos. Se procedió previamente a limpiar las caras del hacha mediante el sistema standard (Grace, 1989, 11-12), sumergiendo ésta en un tanque ultrasónico durante 60 minutos y pasando a continuación un algodón impregnado en alcohol metílico.

Hay estrías corriendo perpendiculares u oblicuas respecto al filo pero aparecen precisamente en la zona donde no hay lustre y por su tamaño se corresponden con las producidas por el pulimento previo del útil (fig. 4,2). El corte está en buen estado aunque algo machacado (crushed) y con algunos lascados. En las zonas donde el lustre estaba más visible a simple vista aparece pulimento, a veces con una estructuración lineal perpendicular al filo (foto K, fig. 5,3; 50x), y en ocasiones se distinguen finas estrías dentro de dicho pulimento entrecruzándose aunque con una orientación genéricamente oblicua respecto al filo (foto D', fig. 5,4; 200x); se aprecian todavía residuos (foto $\mathrm{T}$, fig. 6,1; 200x). Dada la clara persistencia de restos orgánicos se sumerge la pieza en una solución de detergente enzimático en dos períodos de 1 hora cada uno, separados por observaciones. Tras esta limpieza sólo se observa pulimento en las partes más altas de la superficie (foto R', fig. 6,2;100x), disposición que se corresponde más bien con el roce contra un material duro que con madera. En otras palabras, las zonas más claras que se observan serían el resultado del pulimento previo de la pieza, hipótesis que se refuerza al documentar observaciones idénticas en una zona alejada del filo, en pleno centro de ésta (foto $B$, fig. $6,3 ; 100 x)$, a su vez de aspecto muy semejante al que existía en la misma zona con anterioridad a la utilización (fig. 4,2).

\section{B. Análisis microscópico de microhachas}

Las cinco piezas que integran la muestra estudiada tienen procedencias dispares. Dos de ellas (n. ${ }^{1}$ y 2) fueron recuperadas en túmulos megalíticos, la n. .9 proviene, al parecer, de un castro, en tanto que 
las dos últimas carecen de contexto arqueológico. El presente estudio tiene pues un valor meramente indicativo, ya que se manejan artefactos de atribución cultural probablemente diferente y por otro lado carecemos de datos experimentales para contrastar las huellas que puedan detectarse sobre la superficie de las piezas analizadas.

La conservación de todas las piezas era excelente y sólo la pieza n.ำ 1 mostraba a nivel macroscópico algunas fracturas en la zona proximal aunque tanto en ésta como en las restantes el filo se presenta afilado, sin desgaste aparente. En todos los casos se había aplicado un pulimento integral sobre la totalidad de la superficie del objeto.

№ 1.- Depósito: Departamento de Historia I, Universidad de Santiago (fig. 2,1).

Procedencia: mámoa de Rechaba (Tordoia, La Coruña).

Material: sílex. Longitud máxima: $49 \mathrm{~mm}$. Anchura máxima: $27 \mathrm{~mm}$. Espesor máximo: $9 \mathrm{~mm}$. Peso: 19 grs.

CARA A: se señalan estrías largas y profundas corriendo sobre los tercios proximal y medial del objeto (foto A, fig. 6,$4 ; 100 x$ ). Estas estrías desaparecen en el tercio distal para dar paso a otras, más finas, entrecruzándose con frecuencia, entre las que se detectan áreas aisladas de pulimento, dentro de las cuales se distinguen a su vez muchas estrías sin dirección predominante (foto C, fig. 7,1; 200x). Las estrías en la zona activa corren en distintos ángulos respecto al filo (perpendiculares u oblicuas). Por otro lado las estrías finas están presentes también en el tercio proximal, yuxtapuestas sobre las más profundas (foto D, fig. 7,2; 200x), lo cual podría indicar que, lejos de ser las primeras consecuencia de la utilización de la pieza, podría tratarse de las huellas de un último pulimentado fino con agua y arena. CARA B: se repiten las observaciones ya comentadas anteriormente, con la sola diferencia de que aquí las grandes estrías se disponen con más frecuencia paralelamente al filo.

FILO: aparece muy desgastado, particularmente en la cara A, que muestra gran número de lascados, algunos de ellos con las aristas redondeadas.

№ 2.- Depósito: Museu Arqueolóxico de A Coruña.

Procedencia: túmulo n. ${ }^{\circ} 6$ de Os Campiños (Rianxo, La Coruña) (fig. 2,2).

Material: fibrolita. Longitud máxima: $35 \mathrm{~mm}$. Anchura máxima: $22 \mathrm{~mm}$. Espesor máximo: 8’5 mm. Peso: 8 grs.

CARA A: en las inmediaciones del filo se observan estrías muy finas y agrupadas, que en la mitad izquierda corren paralelas al corte, haciéndose progresivamente más oblicuas (foto $\mathrm{B}$, fig. 7,$3 ; 100 \mathrm{x}$ ) hasta que en el extremo derecho son casi perpendiculares. En los tercios medial y proximal hay estrías más gruesas, dispuestas casi paralelamente al filo de la pieza (foto $\mathrm{F}$, fig. 7,4; 100x).

CARA B: se observa el mismo tipo de estrías, pero junto a las finas hay otras gruesas en el área próxima al filo, aunque no hay correspondencia con la pauta de distribución en la otra cara, que es exactamente la contraria. En la zona medial hay estrías corriendo perpendiculares respecto al filo, al igual que en el extremo distal donde también hay algunas paralelas, algunas de éstas son finas pero otras son más gruesas.

FILO: presenta un buen número de lascados que configuran en la cara $\mathrm{A}$ un auténtico escalón, especialmente en la parte media (fig. 7,3).

№ 3.- Depósito: Museo de Pontevedra, reg. 6115.

Procedencia: castro de Santa Cruz (fig. 2,3).

Material: fibrolita. Longitud: $41 \mathrm{~mm}$. Anchura máxima: $24 \mathrm{~mm}$. Espesor máximo: $10 \mathrm{~mm}$. Peso: 15 grs. 
CARA A: hay algunos grupos de estrías finas y también gruesas entrecruzándose sin que se observe una dirección predominante. Esta observación se produce tanto en la zona activa como en la parte medial (foto $\mathrm{E}$, fig. 8,$1 ; 100 x$ ). En la lámina $\mathrm{E}$ puede observarse una estría producida modernamente, yuxtapuesta sobre las antiguas. En la foto B (fig. 8,$2 ; 200 x$ ) se muestra una zona pulimentada que parece ser resultado del contacto con una materia dura (¿metal, hueso?).

CARA B: aquí se señalan las mismas características que en la cara opuesta.

FILO: no está bien terminado, observándose un mayor número de lascados en la cara $\mathrm{B}$ que en la $\mathrm{A}$, todos ellos tienen las aristas redondeadas.

No 4.- Depósito: Museo de Pontevedra, reg. 2865.

Procedencia: San Miguel de Taborda (Tomiño, Pontevedra) (fig. 2,4).

Material: fibrolita. Longitud: $35 \mathrm{~mm}$. Anchura máxima: $20 \mathrm{~mm}$. Espesor máximo: $8 \mathrm{~mm}$. Peso: 6 grs.

CARA A: se señalan algunas grandes estrías corriendo paralelas al filo asi como otras perpendiculares a éste. En el resto del cuerpo sólo se observan unas pocas estrías, con algunos restos de pulimento (foto C, fig. 8,3; 100x).

CARA B: en la zona activa aparecen los mismos elementos: estrías entrecruzándose, algunas zonas con pulimento.

FILO: se conserva bien, con sólo unos escasos lascados.

№ 5.- Depósito: Museo de Pontevedra, reg. 2864.

Procedencia: desconocida (fig. 2,5).

Material: desconocido. Longitud: $34 \mathrm{~mm}$. Anchura máxima: $20 \mathrm{~mm}$. Espesor máximo: $12 \mathrm{~mm}$. Peso: 14 grs.

CARA A: existe en toda la superficie un pulimento brillante y regular, salvo en las depresiones. No se observan estrías excepto en la zona activa, corriendo paralelas al filo (foto A, fig. 8,4; 100x). En la gran acanaladura situada en el lado izquierdo aparecen estrías con una clara orientación longitudinal, probablemente resultantes de la labor de aserrado del útil (foto $C$, fig. 9,1; 100x).

CARA B: tanto en la zona activa como en el extremo proximal aparecen gruesas estrías con una sección en $U$ muy abierta, profundas y generalmente aisladas unas de otras, disponiéndose perpendicular o paralelamente al filo (foto D, fig. 9,2; 200x).

FILO: no se presenta en forma de una arista definida sino como un extremo muy redondeado.

\section{CONCLUSIONES}

Los comentarios que vamos a realizar a continuación deben ser en buena parte valorados con un. alto grado de provisionalidad dado lo limitado de nuestro trabajo experimental y analítico, pero creemos que es oportuno avanzar de forma breve algunas conclusiones o líneas de trabajo futuras a partir de lo ya hecho. En relación con el experimento de tala y subsiguiente estudio microscópico del desgaste se pueden resaltar los siguientes puntos:

A) Un trabajo de tala durante corto tiempo no produjo un nivel significativo de desgaste sobre el filo y zonas adyacentes, tanto a nivel macroscópico como microscópico, en este último caso dentro del umbral de los $200 \mathrm{x}$.

B) El lustre patente después de la utilización en las inmediaciones del corte se distribuía de una forma asimétrica, acorde con la mecánica del uso de un hacha taladora, según Semenov y otros autores. 
Por otro lado, una vez efectuada una limpieza, este lustre desapareció por completo, demostrándose de esa forma que no era consecuencia de un proceso de abrasión de la capa superficial del hacha sino que probablemente se había formado por residuos orgánicos amalgamados por la combinación del calor y presión resultantes del impacto de la zona activa sobre la madera.

C) Existían estrías, a veces dispuestas de forma oblicua, con anterioridad a la utilización de la pieza; asimismo se señalaban algunos lascados previos. Esto hace necesario el establecimiento de criterios discriminantes, basados en experimentación con réplicas actuales, para establecer, si ello es factible, cuáles de esas características son debidas al uso y cuáles al proceso de manufactura. En este mismo sentido es necesario proceder a la observación macro y microscópica de los útiles antes de utilizarlos.

D) A tenor de lo observado en este experimento, es factible que hachas u otro tipo de útiles de corte con poco o ningún desgaste en apariencia, hayan sido sin embargo empleados durante algún tiempo antes de su abandono o deposición ${ }^{3}$.

Como corolario de las observaciones microscópicas realizadas sobre la muestra de microhachas, hay que constatar en primer lugar la existencia en tres de las microhachas de dos tipos distintos de estrías: unas finas y agrupadas y otras más gruesas y aisladas. La explicación de cada uno de estos tipos no es sencilla puesto que ninguno de ellos aparece recluído en una zona concreta del útil, sino que pueden aparecer indistintamente en la zona activa o en el extremo proximal, disponiéndose además con diferentes orientaciones respecto al filo. La conjunción de ambos factores nos lleva a pensar que dichas estrías no son resultado del uso sino de la elaboración o reafilado de las piezas. En experimentos de pulimento de hachas se han documentado estrías muy semejantes a las de tipo más grueso, cuando se utilizaban pulidores a base de arenisca (Madsen, 1984, 56; Meeks, Sieveking, Tite y Cook, 1982, 327). En cuanto a las más finas, podrían deberse a un pulimento empleando un agente abrasivo intermedio como arena y agua, técnica presente en el hacha empleada en el experimento de tala, en cuya superficie se observaban con anterioridad a dicho trabajo estrías de estas características (fig. 4,2). Por otro lado, el estado de los filos apunta hacia la posibilidad de un enmangue -y consecuente utilización- transversal, de tipo azuela, evidenciado por el desgaste diferencial en el corte de tres piezas (n. ${ }^{\circ} 1-3$ ), que muestra un mayor número de lascados sobre una de las caras de la zona activa. Estas mismas piezas son clasificables como azuelas asimismo desde el punto de vista morfotipológico. Finalmente, el fortísimo desgaste apreciable micróscopicamente en la microhacha n.. 5 sugiere una utilización intensa que produjo una abrasión del filo, pero no un número apreciable de fracturas. Esta clase de huellas de uso sería más bien consistente con una dinámica de trabajo del tipo que Leroi-Gourhan $(1984,45)$ denomina "percusión lineal apoyada", propia, por ejemplo, de un raspador o cepillo empleados sobre una materia de dureza media.

3. Una idea semejante es expuesta por Crabtree: The obtuse angle as a functional edge, Experimental Archaeology, 1977, págs. 46-47 (citado en Olausson, 1982, 58). 


\section{BIBLIOGRAFIA}

BECKER, C.J. (1949): "Hafted Neolithic Celts II. With observations on a new Funnel-Beaker Type from New Zealand", Acta Archaeologica 20: 231-248.

BORN, P.L. (1971): "Adze wear patterns", Newsletter Missouri Archaeological Society 250: 2-5.

CAUVIN, J. (1968): "Les outillages néolithiques de Byblos et du littoral libanais", Fouilles de Byblos 4, París.

DELIBES DE CASTRO, G. (1974): "Contribución al estudio de las funciones del hacha pulimentada. Resultados de la aplicación del sistema Semenov a 130 ejemplares de Tierra de Campos", Zephyrus 25: 151-154.

DETEV, P. (1960): "Essai d'etablir une distinction entre les haches et les ciseaux, les herminettes, les houes et les socs de l'epoque neolithique", Annuaire du Musée National Archéologique du Plovdiv 4: 61-74.

FABREGAS VALCARCE, R. (1988): "La industria lítica pulimentada", Aproximaciones a la cultura material del megalitismo gallego (R. Fábregas y F. de la Fuente): 15-53.

(1991): Estudio de los ajuares líticos de las sepulturas megalíticas de Galicia y Norte de Portugal, Tesis Doctoral microfilmada, U.N.E.D., Madrid.

GRACE, R. (1989): Teach yourself microwear analysis: A guide to the interpretation of the function of stone tools, Arqueohistórica 3. Santiago de Compostela.

HARDING A. y YOUNG, R. (1979): "Reconstruction of the hafting methods and function of stone implements", Stone Axe Studies (eds. T.H. McK. CLOUGH y W.A. CUMMINS), C.B.A. Research Report 23: 102-105.

HARDING, F. (1983): "An experiment to produce a ground flint axe", The Human Uses of Flint and Chert (eds. G. SIEVEKING y M.H. NEWCOMER). Papers presented at the $4^{\text {th }}$ International Flint Symposium, Brighton: $37-42$.

IVERSEN, J. (1956): "Forest clearance in the Stone Age", Scientific American 194-3: 36-41.

JøRGENSEN, S. (1985): Tree-Felling in Draved, Copenhague.

KANTMAN, S. (1969-70): "A microanalytic study of some ground stone artefacts from Tilmen Höyük and Gedïklï-Karahöyük (Southeastern Anatolia). Contributions to functional typology", Anatolica 3: 139-145.

LEROI-GOURHAN, A. (1984): Evolucâo e técnicas. I - O homem e a matéria, Lisboa.

MADSEN, B. (1984): "Flint axe manufacture in the Neolithic: Experiments with Grinding and Polishing of ThinButted Flint Axes", Journal of Danish Archaeology 3: 47-62.

MEEKS, N.D., SIEVEKING, G. de G., TITE, M.S. y COOK, J. (1982): "Gloss and Use-wear Traces on Flint Sickles and Similar Phenomena", Journal of Archaeological Science, 9: 317-340.

MÜLLER-BECK, H. (1965): Seeberg Burgäschisee-Süd. Holzgeräte und Holzbearbeitung, Acta Bernensia 2-5.

OLAUSSON, D.S. (1982): "Lithic technological analysis of the thin-butted flint axe", Acta Archaeologica 53: $1-87$.

(1982-1983): "Flint and groundstone axes in the Scanian Neolithic. An evaluation of raw materials based on experiment", Scripta Minora 2: 7-66.

QUENTE, P. (1914): "Steinzeitliche Ackerbaugeräte aus der Ostprignitz, Erdhacken und Pflüge, und ihre Schaftungsmöglichkeit", Praehistorische Zeitschrift 6: 180-187.

ROODENBERG, J.J. (1982): "Traces d'utilisation sur les haches polies de Bouqras (Syrie)", Traces d'utilisation sur les outils néolithiques du Proche Orient (ed. M.C. CAUVIN), Travaux de la Maison de l'Orient 5: 177-188.

SEHESTED, N.F.B. (1884): Praktiske forsøg. Archaeologiske Undersøgelser 1878-1881. Copenhague.

SEMENOV, S.A. (1981): Tecnología Prehistórica, Madrid.

SILLITOE, P. (1979): "Stone versus steel”, Mankind 12: 151-161.

SONNENFELD, J. (1962): "Interpreting the function of primitive implements", American Antiquity 28-1: 56-65.

STELCL J. y MALINA, J. (1970): "Anwendung der Petrographie in der Archäeologie”, Folia Facultatis Scientiarum Naturalium Universitatis Purkynianae Brunensis, Geologia 11-5. 

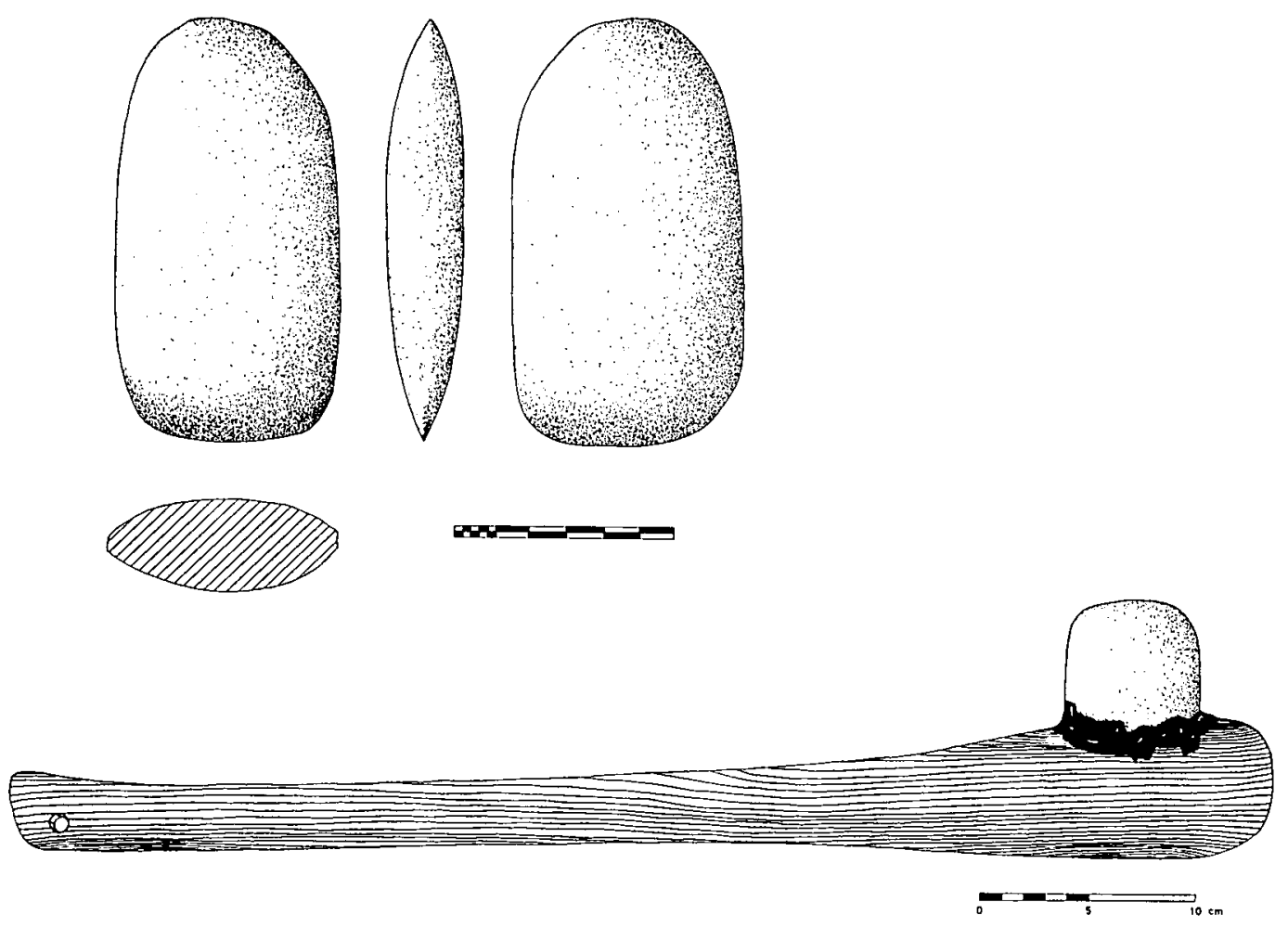

Fig. 1
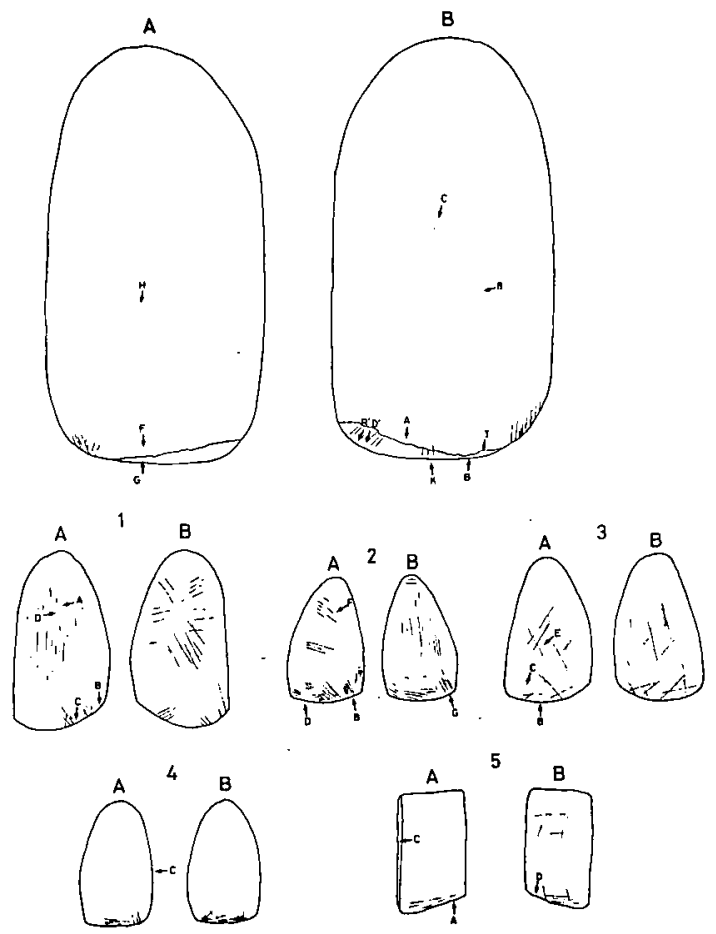

Fig. 2 

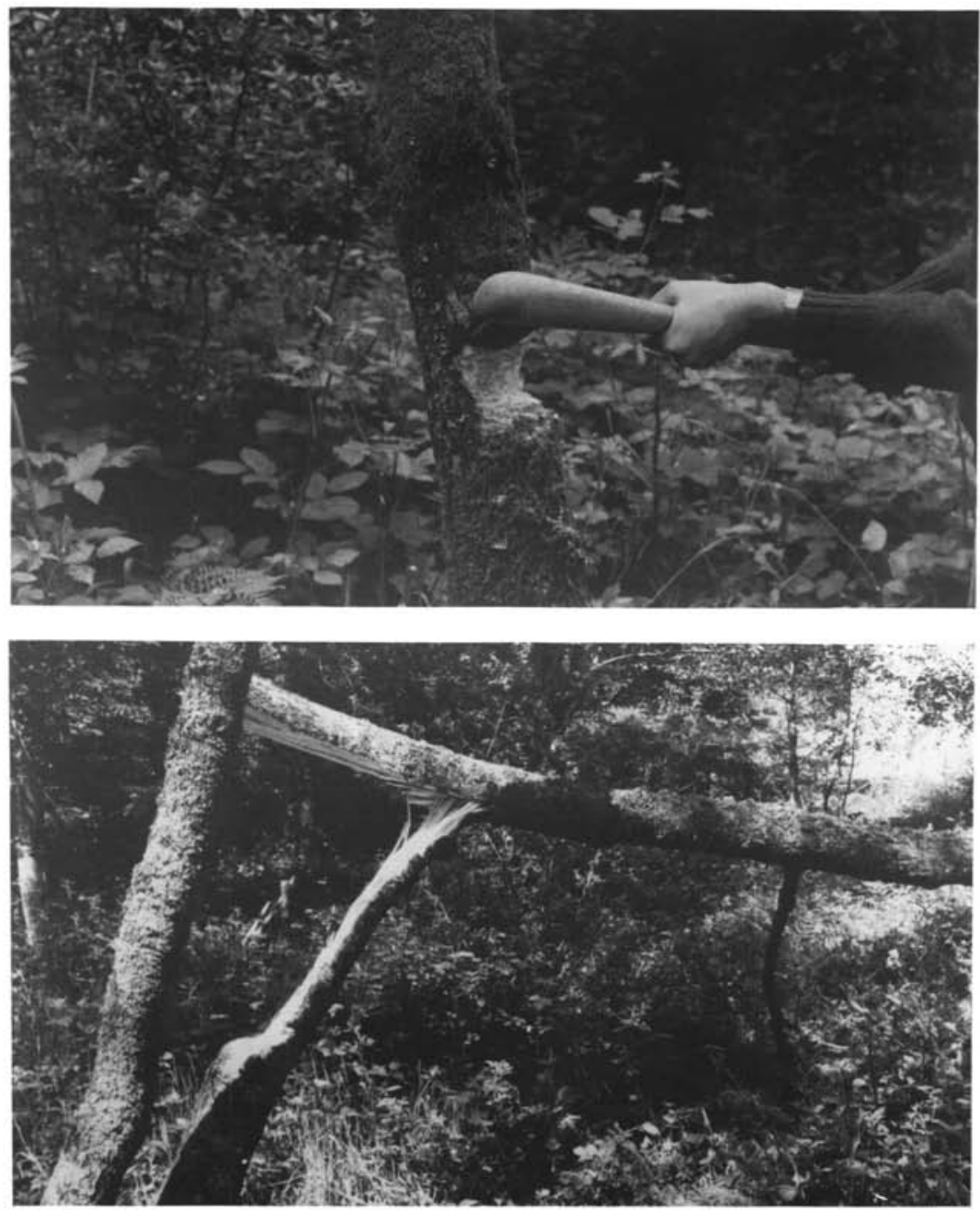

Fig. 3 

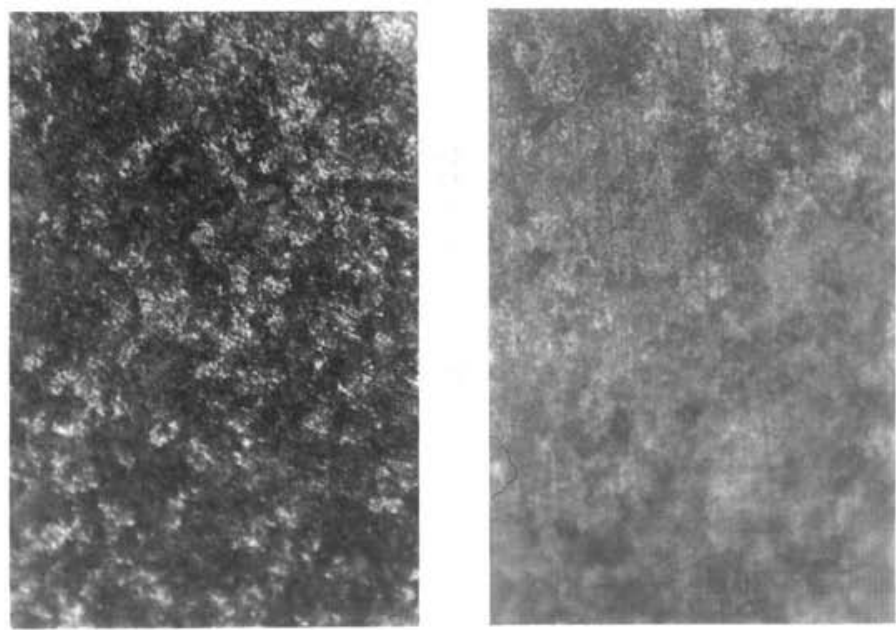

送

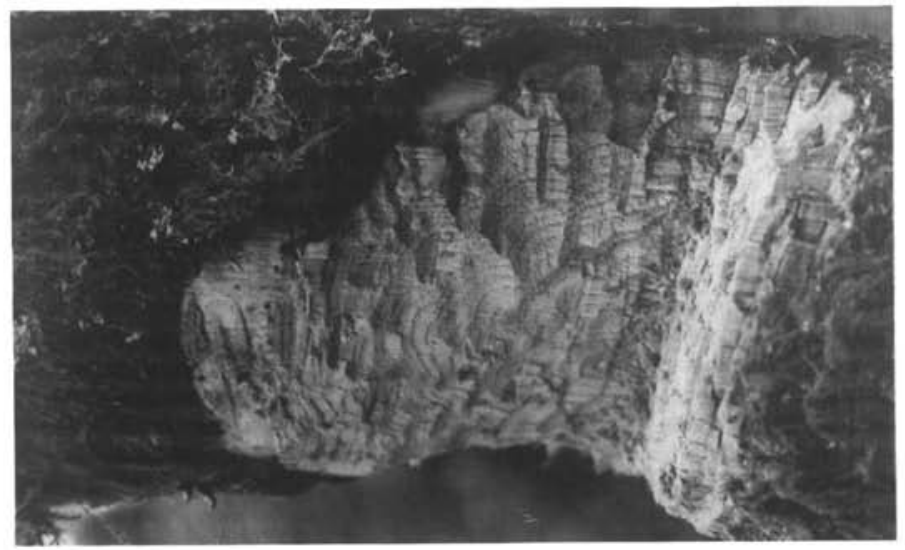



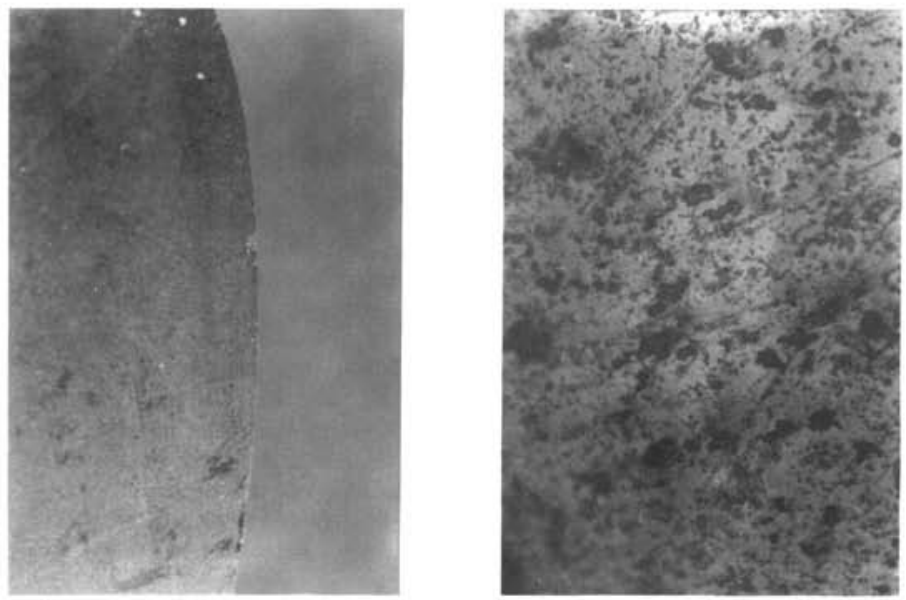

$n$
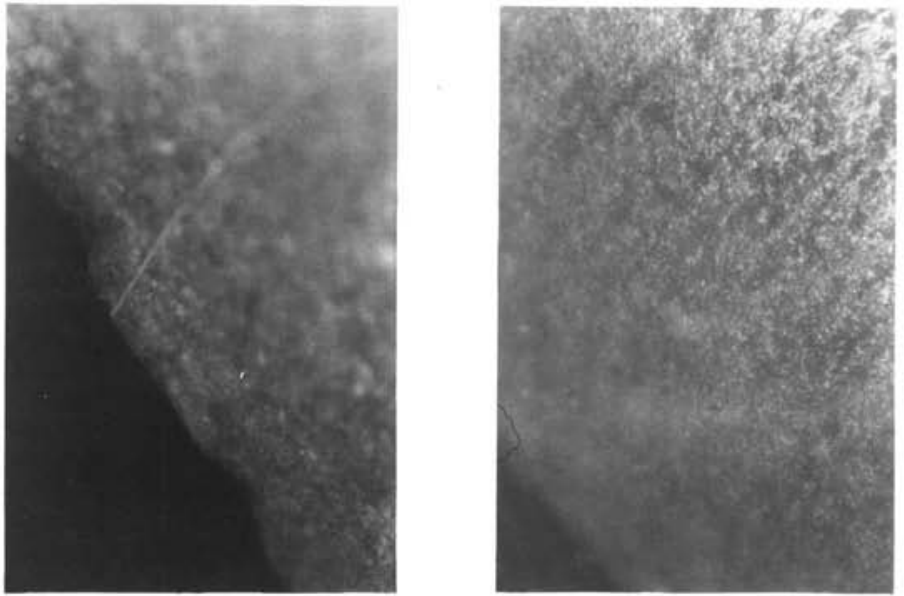

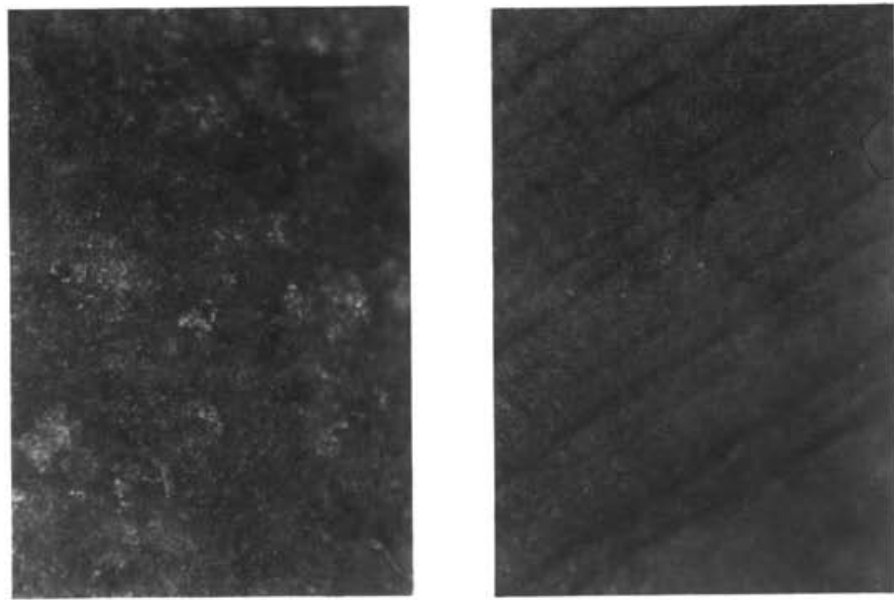

$\stackrel{0}{2}$
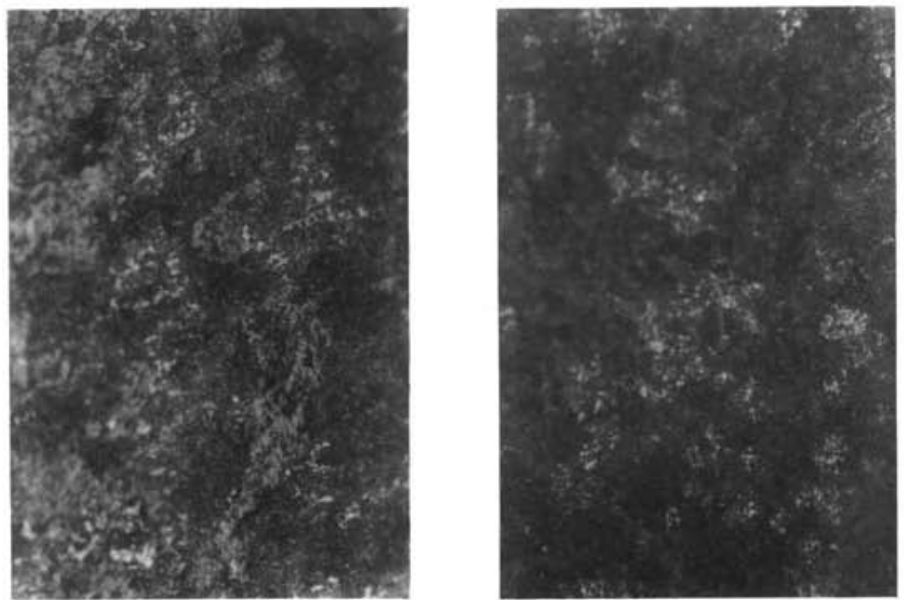

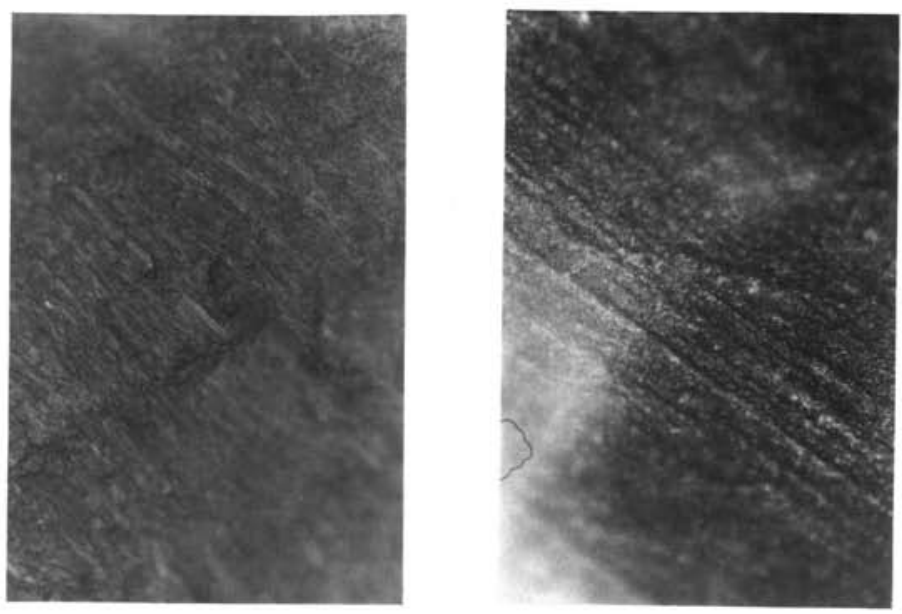

$\hat{2}$
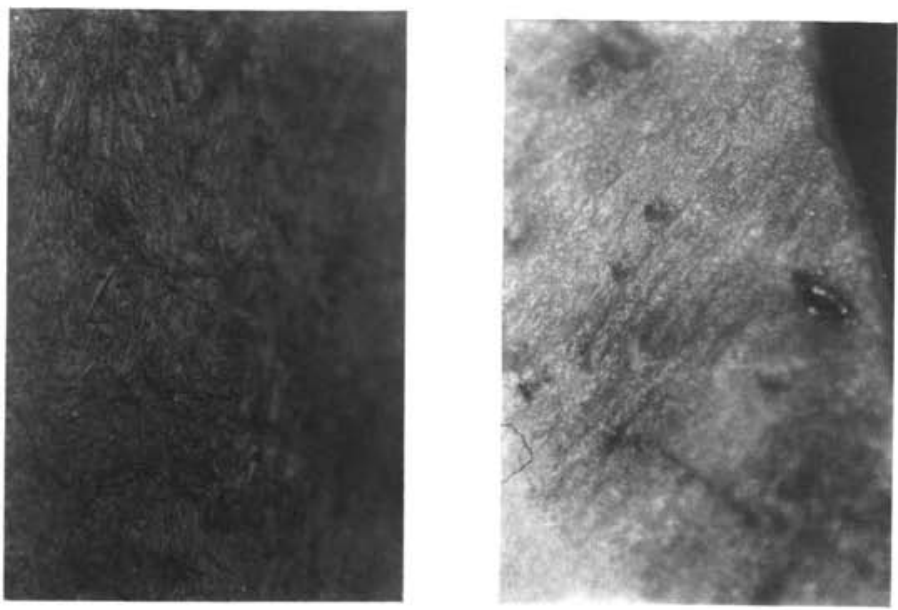

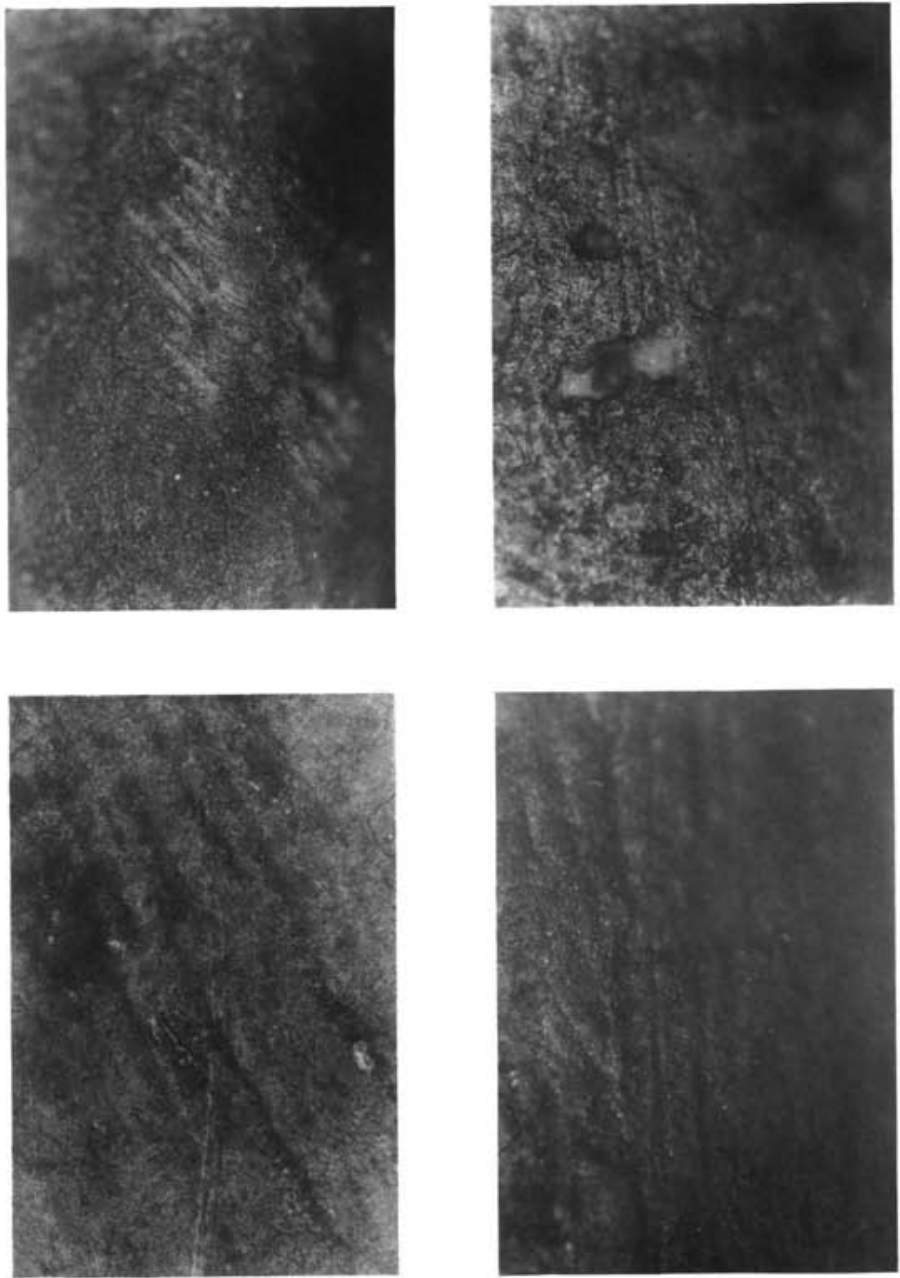

延 

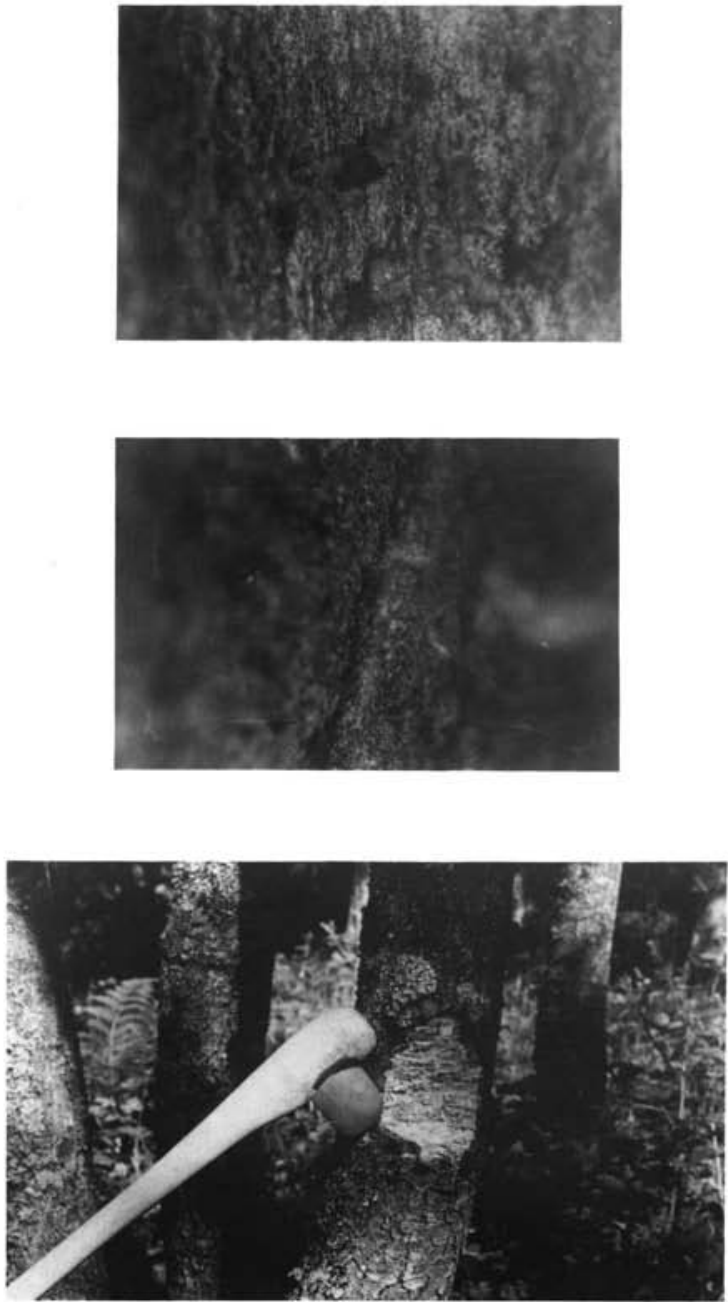

Fig. 9 